\title{
Site-directed mutagenesis reveals a unique requirement for tyrosine residues in IL-7Ra and TSLPR cytoplasmic domains in TSLP-dependent cell proliferation
}

\author{
Jun Zhong, Akhilesh Pandey*
}

\begin{abstract}
Background: Thymic stromal lymphopoietin (TSLP) is an interleukin-7 (IL-7) like cytokine, which plays an important role in the regulation of immune responses to allergens. TSLP binds to a heterodimeric receptor complex composed of the IL-7 receptor $\alpha$ chain (IL-7R $\alpha$ ) and the TSLP receptor (TSLPR, also known as CRLF2). It has previously been suggested that the lone tyrosine residue in the mouse TSLPR cytoplasmic domain is required for cell proliferation using chimeric receptor systems. Also the role of tyrosine residues in the IL-7R $\alpha$ cytoplasmic domain in TSLP signaling has not yet been investigated. We undertook a systematic analysis to test the role of tyrosine residues of both the IL-7R $\alpha$ and the TSLPR in inducing cell proliferation in a growth factor dependent cell line, $\mathrm{Ba} / \mathrm{F3}$.
\end{abstract}

Results: A multiple sequence alignment of the IL-7R $\alpha$ and TSLPR cytoplasmic domains revealed conservation of most, but not all, cytoplasmic tyrosine residues across several species. Our site-directed mutagenesis experiments revealed that the single tyrosine residue in human TSLPR was not required for TSLP-dependent cell proliferation. It has previously been reported that Y449 of human IL-7R $\alpha$ is required for IL-7 dependent proliferation. Interestingly, in contrast to IL-7 signaling, none of tyrosine residues in the human IL-7R $\alpha$ cytoplasmic domain were required for TSLP-dependent cell proliferation in the presence of a wild type TSLPR. However, the mutation of all cytoplasmic four tyrosine residues of human IL-7R $\alpha$ and human TSLPR to phenylalanine residues abolished the proliferative ability of the TSLP receptor complex in response to TSLP.

Conclusion: These results suggest that TSLP requires at least one cytoplasmic tyrosine residue to transmit proliferative signals. Unlike other members of IL-2 cytokine family, tyrosine residues in IL-7R $\alpha$ and TSLPR cytoplasmic domains play a redundant role in TSLP-mediated cell growth.

\section{Background}

Thymic stromal lymphopoietin (TSLP) was first identified as a growth factor in the conditioned medium supernatant from the Z210R.1 thymic stromal cell line to support B cell proliferation in vitro $[1,2]$. TSLP is now known to play a key role in the initiation of asthma $[3,4]$. TSLP shares IL-7R $\alpha$ with IL-7. TSLP signaling is mediated by a heterodimeric receptor complex, which consists of the interleukin-7 receptor $\alpha$ chain (IL-7R $\alpha$ )

* Correspondence: pandey@jhmi.edu

McKusick-Nathans Institute of Genetic Medicine and Departments of Biological Chemistry, Oncology and Pathology, Johns Hopkins University School of Medicine, 733 N Broadway, Baltimore, Maryland, 21205, USA and a unique TSLP-binding receptor (TSLPR), to transmit proliferative signals in cells [5-7]. IL-7 binds to a heterodimeric receptor complex, the IL-7R $\alpha$ and the cytokine receptor common gamma chain $\left(\gamma_{c}\right)$, which is shared by IL-2, 4, 7, 9, 15 and 21. Both TSLP and IL-7 can activate the transcription factor STAT5. In the IL-7 receptor complex, IL-7R $\alpha$ binds to Jak1 and $\gamma_{c}$ binds to Jak3 upon addition of IL-7. However, none of Jak kinases have been reported to be phosphorylated by the binding of TSLP to its receptor [2]. Previous studies showed that Y449 in the IL-7R $\alpha$ cytoplasmic domain provides a docking site for PI-3K and STAT5 and is required for a proliferative signal by IL-7 signaling 
[8-10]. Further, the tyrosine residues of $\gamma_{c}$ are not required for IL-7-mediated cell proliferation [11]. Isaksen and colleagues observed that the lone tyrosine residue of the mouse TSLPR cytoplasmic domain is required for TSLP-mediated cell proliferation using chimeric receptors composed of the human GM-CSFR $\alpha$ chain extracellular domain fused to the mouse TSLPR transmembrane and cytoplasmic domains and the human GM-CSFR $\beta$ chain extracellular domain fused to the mouse IL-7R $\alpha$ transmembrane and cytoplasmic domains [12]. Brown et al. showed that anti-IL-7R $\alpha$ antibodies inhibited TSLP-mediated proliferation of pre B-leukemia [13], indicating that both IL-7R $\alpha$ and TSLPR contribute to TSLP-dependent cell proliferation.

We aligned the protein sequences of IL-7R $\alpha$ and TSLPR cytoplasmic domains and observed that Y449 and Y456 of human IL-7R $\alpha$ and Y368 of human TSLPR were conserved across the species examined while Y401 of human IL-7R $\alpha$ was not conserved. Because the role of tyrosine residues in the context of the 'native' form TSLP receptor complex in TSLP-mediated cell proliferation has not been previously investigated, we took a systematic approach to evaluate the role of cytoplasmic tyrosine residues of TSLP receptor complex in mediating TSLP-induced cell proliferation. Our data show that the cytoplasmic tyrosine residues of either human IL$7 R \alpha$ or human TSLPR can mediate TSLP-induced cell proliferation and that mutation of all the four cytoplasmic tyrosine residues of human IL-7R $\alpha$ and human TSLPR to phenylalanine residues is required to abolish TSLP-dependent cell proliferation.

\section{Results and Discussion}

Most, but not all, cytoplasmic tyrosine residues of IL-7R $\alpha$ and TSLPR are conserved across species

Tyrosine residues in cytokine receptor cytoplasmic domains play important roles in mediating cytokine signaling and are mostly conserved across species. We aligned the amino acid sequences of IL-7R $\alpha$ and TSLPR cytoplasmic domains from various species. Figure $1 \mathrm{~A}$ shows an alignment of the IL-7R $\alpha$ cytoplasmic domains. The membrane-proximal domains and C-terminal regions harboring two tyrosine residues are conserved among all IL-7R $\alpha$ chains while the regions in between vary to some extent. The IL-7R $\alpha$ cytoplasmic domains in Mus musculus, Rattus norvegicus, and Gallus gallus have four tyrosine residues while the IL-7R $\alpha$ cytoplasmic domains in Homo sapiens, Pan troglodytes, Macaca mulatta, Canis lupus familiaris and Bos taurus only harbor three tyrosine residues. As indicated by red arrows in Figure 1A, the two tyrosine residues in the C-terminal region of the IL-7R $\alpha$ chains are conserved while other tyrosine residues in the variable region are not conserved (red triangles). Figure 1B shows an alignment of the TSLPR cytoplasmic domains. As observed in the case of IL-7R $\alpha$, the membrane-proximal domains and $\mathrm{C}$-terminal regions harboring one tyrosine residue are conserved in all TSLPRs while the intervening regions are variable. TSLPR cytoplasmic domains in Homo sapiens, Mus musculus, Rattus norvegicus and Canis lupus familiaris have only one tyrosine residue while the TSLPR cytoplasmic domain in Bos taurus harbors two tyrosine residues. Interestingly, the tyrosine residue in the C-terminal region of the TSLPR cytoplasmic domains across species is also conserved (a red arrow in Figure 1B). Because tyrosine residues play an important role in mediating the signaling by cytokine receptors and are conserved in the IL-7R $\alpha$ and TSLPR cytoplasmic domains, we wanted to examine the role of these tyrosine residues in TSLP-dependent cell proliferation.

The lone cytoplasmic tyrosine residue in TSLPR is not required for TSLP-dependent cell proliferation

Cytokines IL-2, 4, 7, 9, 15 and 21 share the common receptor subunit $\gamma_{c}$ that shows high homology to TSLPR. It has been reported earlier that these cytokines do not require the tyrosine residues of the $\gamma_{c}$ cytoplasmic domain to support cell growth [11]. In contrast, Isaksen and colleagues reported earlier that the single tyrosine residue of the mouse TSLPR cytoplasmic domain is critical for TSLP-dependent cell proliferation [12]. Because the study was based on a chimeric receptor system, we sought to study the role of tyrosine residues in TSLP signaling in the context of the native TSLP receptor complex. TSLP requires the heterodimeric TSLP receptor complex - IL-7R $\alpha$ and TSLPR - to transmit signals (Figure 2A). Reche et al. have shown that coexpressed human TSLPR and IL-7R $\alpha$ receptor subunits respond to human but not mouse TSLP [7]. We retested the requirement of the receptor complex for human TSLP-mediated signaling in an IL-3 dependent mouse cell line, $\mathrm{Ba} / \mathrm{F} 3$, which also expresses endogenous murine TSLPR. A retroviral system was used to generate $\mathrm{Ba} / \mathrm{F} 3$ cells that express wild type hTSLPR and/or wild type hIL-7R $\alpha$. As shown in Figure 2B, only $\mathrm{Ba} / \mathrm{F} 3$ cells expressing both hIL-7R $\alpha$ and hTSLPR, but not those expressing hIL-7R $\alpha$ or hTSLPR alone could proliferate in response to human TSLP. Cell surface expression of human TSLPR and human IL-7R $\alpha$ was confirmed by flow cytometry analysis using anti-human TSLPR and anti-human IL-7R $\alpha$ antibodies (Figure 2C). These results again confirmed the requirement of hIL$7 R \alpha$ and hTSLPR for human TSLP action allowing us to use this system for a systematic analysis of the requirement of tyrosine residues.

Human TSLPR contains only one cytoplasmic tyrosine residue (Y368) very close to the carboxyl terminus (Figure 1A). To determine whether this residue is required for TSLP-mediated cell proliferation, it was 


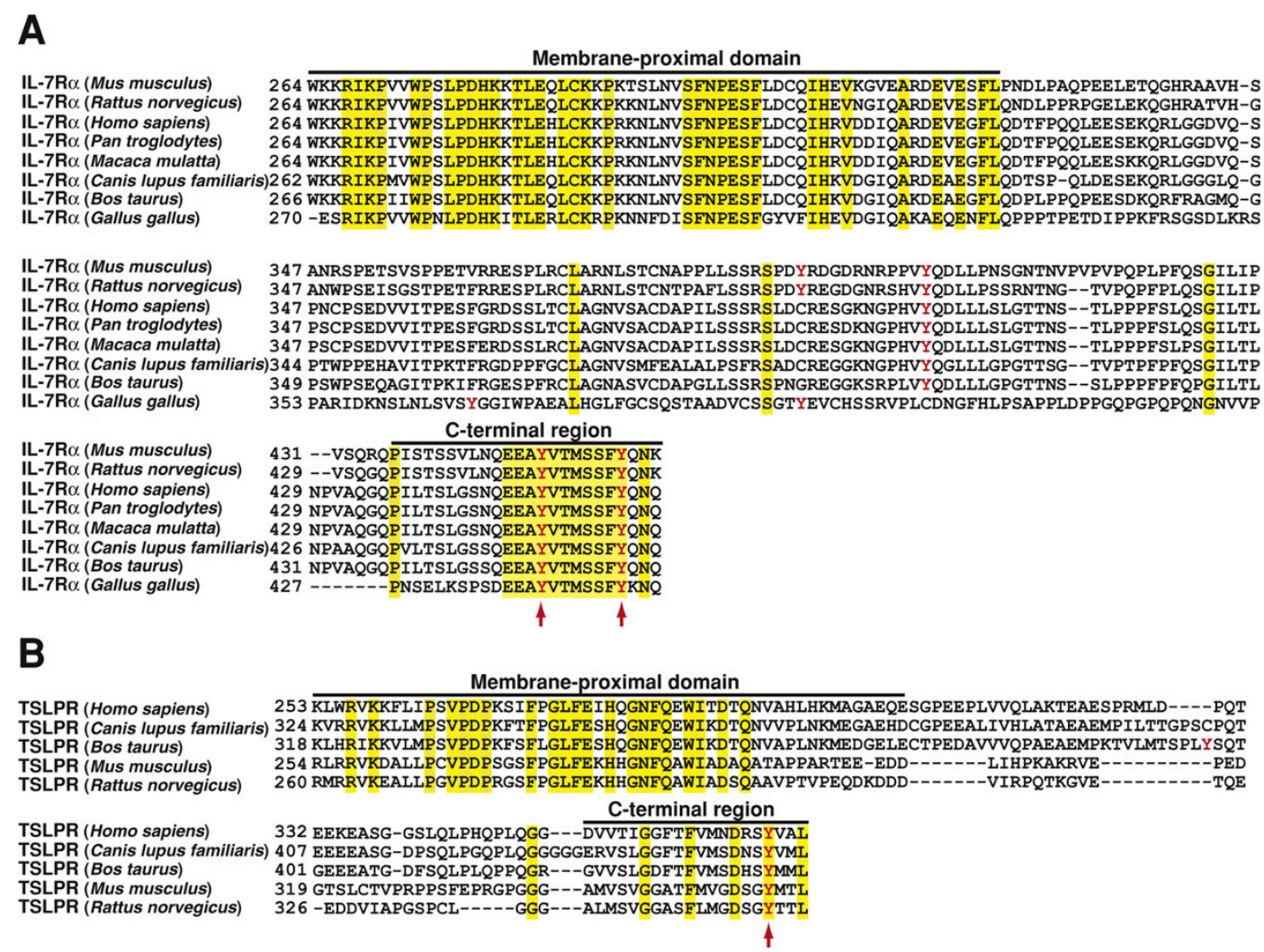

Figure 1 Conservation of cytoplasmic tyrosine residues in IL-7R $\alpha / T S L P R$ across species. Sequence alignments of (A) the IL-7R $\alpha$ cytoplasmic domains from Mus musculus, Rattus norvegicus, Homo sapiens, Pan troglodytes, Macaca mulatta, Canis lupus familiaris, Bos taurus, and Gallus gallus and (B) the TSLPR cytoplasmic domains from Homo sapiens, Canis lupus familiaris, Bos taurus, Mus musculus, and Rattus norvegicus were performed using ClustalX version 2.0.10 with the BLOSUM62 matrix. The conserved amino acid residues are highlighted in yellow while tyrosine residues are in red; conserved tyrosine residues are indicated by a red arrow.

replaced by a phenylalanine residue $(\mathrm{Y} 368 \mathrm{~F})$. A Ba/F3 cell line expressing both hTSLPR (Y368F) and hIL7R $\alpha$ (WT) was established using retrovirus-based infection. As shown in Figure 2D and 2E, mutation of this tyrosine residue failed to abolish the proliferative response to TSLP. On day 3 of culture, $100 \mathrm{ng} / \mathrm{ml}$ TSLP induced $\sim 25 \%$ more proliferation in $\mathrm{Ba} / \mathrm{F} 3$ cells expressing hTSLPR (Y368F)/hIL-7R $\alpha$ (WT) than Ba/F3 cells expressing hTSLPR (WT)/hIL-7R $\alpha$ (WT) (p-value < 0.01) (Figure 2D). To examine this in greater detail, we carried out a dose response study (Figure 2E). We observed that in contrast to what was observed at 100 $\mathrm{ng} / \mathrm{ml}, \mathrm{Ba} / \mathrm{F} 3$ cells expressing the hTSLPR (Y368F)/hIL$7 \mathrm{R} \alpha(\mathrm{WT})$ pair grew at a slower rate $(\mathrm{p}$-value $<0.01)$ than $\mathrm{Ba} / \mathrm{F} 3$ cells expressing the hTSLPR (WT)/hIL-7R $\alpha$ (WT) in response to low doses of TSLP $(1 \mathrm{ng} / \mathrm{ml}$ and $10 \mathrm{ng} / \mathrm{ml}$ ). There was no statistical difference between the two at $0.01 \mathrm{ng} / \mathrm{ml}$ and $0.1 \mathrm{ng} / \mathrm{ml}$. The cell surface expression of human TSLPR and human IL-7R $\alpha$ was similar across the cell lines as confirmed by FACS using anti-human TSLPR and anti-human IL-7R $\alpha$ antibodies (data not shown). Nevertheless, in contrast to a previous report using a chimeric receptor system [12], our data showed that the lone tyrosine residue in the TSLPR cytoplasmic domain is not required for TSLP-dependent cell proliferation. Our findings suggest that studies about TSLP-mediated signaling pathways should be carried out in the context of the native TSLP receptor complex. Intriguingly, these data suggest that Y368 in the hTSLPR cytoplasmic domain may play an inhibitory role in TSLP signaling in response to high doses of TSLP while playing a positive role at lower doses.

The cytoplasmic tyrosine residues in IL-7R $\alpha$ are required for IL-7 but not TSLP-dependent cell proliferation

Like TSLP, the receptor complexes for IL-4, 7, 9 and 21 are all heterodimers. Previous studies showed that Y497 of the human IL-4R is essential for IL-4 mediated proliferation [14] and that Y449 of the human IL-7R $\alpha$ is required for IL-7 induced cell growth $[8,9]$. It has also been shown that IL-9 requires Y407 of the IL-9R to transmit proliferative signals and activate STATs [15] and that Y501 of the IL-21R is critical for maximal IL-21-mediated proliferation [16]. The human IL-7R $\alpha$ contains three tyrosine residues in its cytoplasmic 


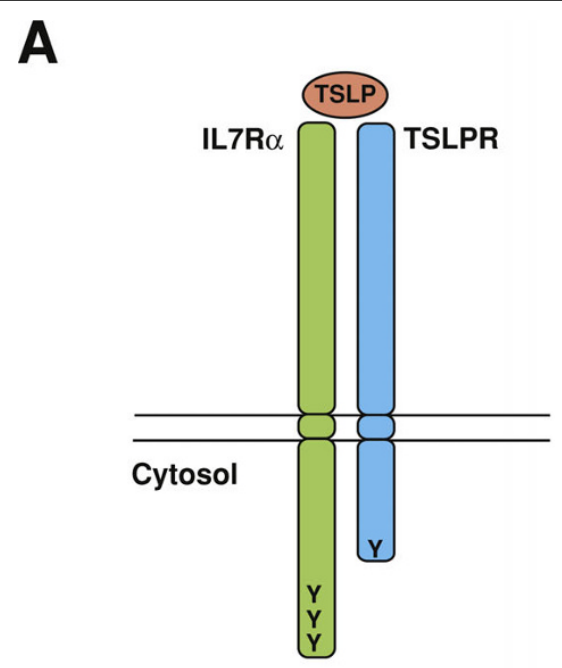

B

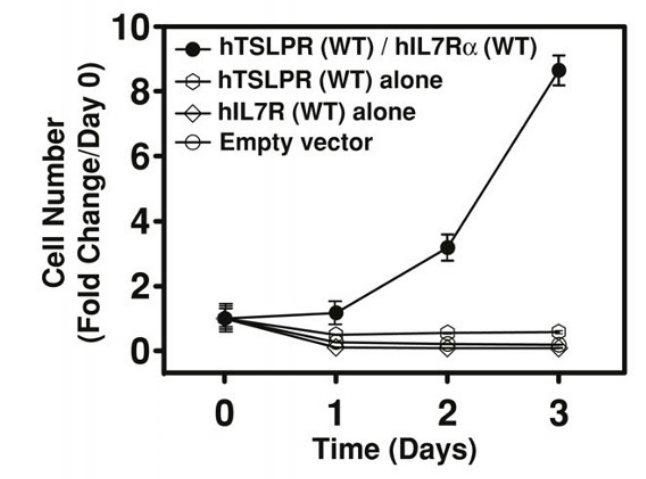

C

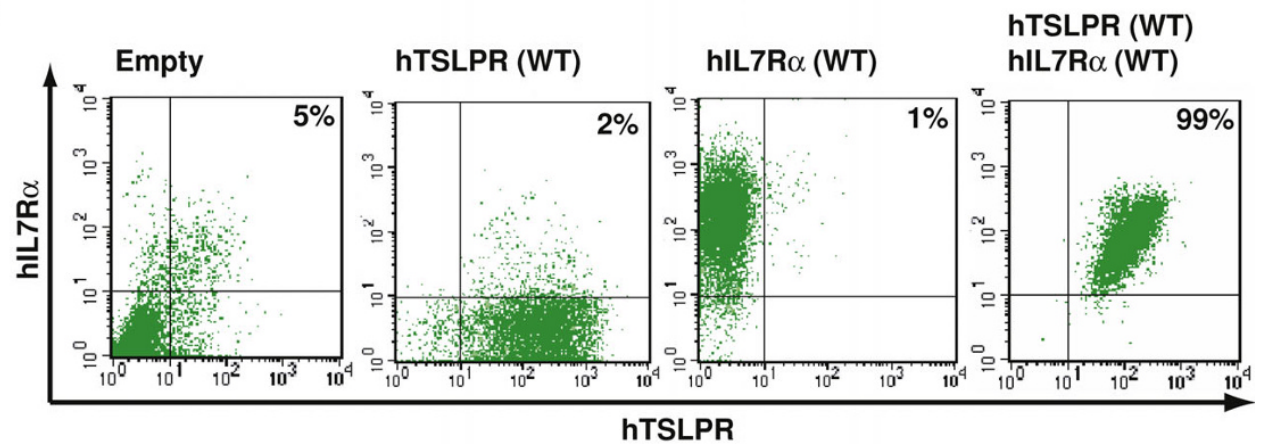

D

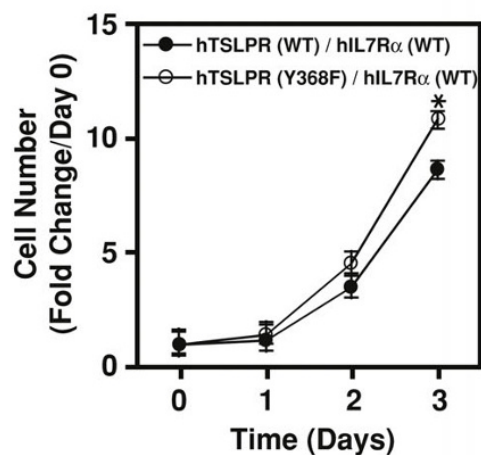

E

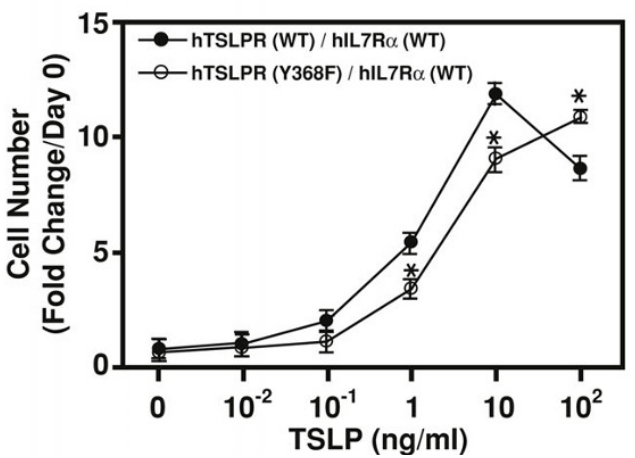

Figure 2 The lone cytoplasmic tyrosine residue of human TSLPR is not required for TSLP-dependent cell proliferation. (A) A schematic illustration of the human TSLP receptor complex composed of the human IL-7R $\alpha$ and the human TSLPR. Y denotes the cytoplasmic tyrosine residues. (B) Exponentially growing Ba/F3 cells were starved and then treated with human TSLP (100 ng/ml). The data are represented as fold changes with the number of cells seeded on day 0 (for TSLP stimulation) representing the baseline. The mean of experiments done in triplicate for each time point and each cell line is shown. Error bars indicate S.E.M. (C) Surface expression of the wild type human TSLPR and IL-7R $\alpha$ in the cell lines used in (B). Exponentially growing infected Ba/F3 cells were analyzed by flow cytometry for cell surface expression of human TSLPR and IL-7R $\alpha$. (D) TSLP-induced proliferation of Ba/F3 cells co-expressing the indicated were examined as described in (B). Statistical significance of the proliferative responses in the different cells at the same time point was evaluated using unpaired Student's t-test ${ }^{*}$ indicates a significant difference with $p$-value $<0.01$ ). (E). Exponentially growing Ba/F3 cells were starved and then stimulated with the indicated concentration of human TSLP for 3 days. Cell numbers were counted after 3 days. The data are represented as fold changes with the number of cells seeded on day 0 (for TSLP stimulation) representing the baseline. Statistical significance of the proliferative responses in the different cells in response to the same dose of TSLP was evaluated using unpaired Student's t-test (* indicates a significant difference with $p$-value $<0.01$ ). (The growth of cells was examined in 3 independent sets of experiments and found to be similar. The results from one representative experiment are shown). 
domain. Because the cytoplasmic tyrosine residue of human TSLPR is not required for cell proliferation and cytoplasmic tyrosine residues are critical for cytokine receptor signaling, we wanted to determine the requirement of the tyrosine residues in the IL-7 $\alpha$ cytoplasmic domain for TSLP-dependent cell proliferation. We replaced the cytoplasmic tyrosine residues in IL-7R $\alpha$ with phenylalanine residues to generate IL-7R $\alpha$ mutants. First, we generated three individual mutants of human IL-7R $\alpha$ - Y401F, Y449F and Y456F. Three Ba/F3 cell lines expressing the wild type TSLPR along with the three mutated IL-7R $\alpha$ were established using retrovirusbased infection. Interestingly, none of the three mutations in the human IL-7R $\alpha$ cytoplasmic domain abolished the proliferative response to TSLP administration (Figure 3). The individual mutations, Y401F (Figure 3A), Y449F (Figure 3C) and Y456F (Figure 3E), even led to an enhanced proliferative response at $100 \mathrm{ng} / \mathrm{ml}$ of TSLP. However, at lower doses of TSLP, Y449F (Figure 3D) and Y456F (Figure 3F), exhibited a diminished response ( $\mathrm{p}$-value $<0.01$ ). Because the three tyrosine residues in hIL-7R $\alpha$ may play redundant roles in supporting cell proliferation, we mutated all of them to phenylalanine residues (Y401F/Y449F/Y456F) and transduced this mutant receptor into $\mathrm{Ba} / \mathrm{F} 3$ cells along with wild type human TSLPR. Strikingly, the mutation of all three cytoplasmic tyrosine residues of the human IL-7R $\alpha$ to phenylalanine residues was still unable to abolish the proliferative response to TSLP (Figure 3G and $3 \mathrm{H})$. The cell surface expression of mutant or wild type hIL-7R $\alpha$ and hTSLPR was similar as evidenced by FACS (data not shown).

Because the role of tyrosine residues in human IL-7R $\alpha$ cytoplasmic domain in proliferation induced by IL-7, which requires IL-7R $\alpha$ and $\gamma_{c}$ (Figure 4A), was established in a chimeric receptor system $[8,9]$, we revisited this issue in $\mathrm{Ba} / \mathrm{F} 3$ cells. Ba/F3 cells expressing the wild type or mutant human IL-7R $\alpha$ (Y401F/Y449F/Y456F) and the wild type human $\gamma_{\mathrm{c}}$ were established and evaluated for cell proliferation upon addition of human IL-7. In contrast to what we observed with TSLP, mutation of the three tyrosine residues in IL-7R $\alpha$ completely abolished $\mathrm{Ba} / \mathrm{F} 3$ cell proliferation in response to IL-7 (Figure 4B), which is consistent with previous reports $[8,9]$. Cell surface expression of human IL-7R $\alpha$ and human $\gamma_{c}$ were similar by FACS using anti-human IL-7R $\alpha$ and anti-human $\gamma_{c}$ antibodies (Figure 4C). Taken together, our data show that the cytoplasmic tyrosine residues in IL-7R $\alpha$ are required for IL-7 but not TSLP-dependent cell proliferation.

TSLP-dependent cell proliferation requires the presence of at least one tyrosine residue

We next wanted to test whether any combination of mutation of tyrosine residues in IL-7R $\alpha$ or TSLPR could affect TSLP-induced cell proliferation. For this, we generated three additional cell lines, hTSLPR (Y368F)/hIL$7 \mathrm{R} \alpha$ (Y401F), hTSLPR (Y368F)/hIL-7R $\alpha$ (Y449F) and hTSLPR (Y368F)/hIL-7R $\alpha$ (Y456F). As shown in Figure $5 \mathrm{~A}, \mathrm{C}$ and $5 \mathrm{E}$, none of these combinations abolished cell proliferation in response to TSLP. As observed previously for individual tyrosine residues in IL-7R $\alpha$, combining them with TSLPR tyrosine mutants still led to a diminished response at low doses of TSLP (p-value $<0.01$ ) (Figure 5B, D and 5F).

To test if any tyrosine residues were required for TSLP-induced proliferation, we mutated all tyrosine residues in the TSLP receptor complex (TSLPR (Y368F)/IL-7R $\alpha$ (Y401F/Y449F/Y456F)). As shown in Figure $5 \mathrm{G}$ and $5 \mathrm{H}$, this abolished the ability of the human TSLP receptor complex to drive cell proliferation. Taken together, our data suggest that TSLP signaling requires at least one tyrosine residue to support cell growth while all the four tyrosine residues in human TSLP receptor complex play various roles in TSLP-mediated cell proliferation.

STAT5 phosphorylation requires at least one cytoplasmic tyrosine residue in the human TSLP receptor complex

Both human and mouse TSLP can induce STAT5 phosphorylation $[2,7]$. We examined whether TSLP-induced phosphorylation of STAT5 requires any of the cytoplasmic tyrosine residues of human TSLP receptor complex. As shown in Figure 6, while TSLP induced STAT5 phosphorylation in cells expressing wild type receptors and those expressing hTSLPR (WT)/hIL7R $\alpha$ (Y401F/ Y449F/Y456F), no phosphorylation was detectable in cells expressing hTSLPR (Y368F)/hIL7R $\alpha$ (Y401F/ Y449F/Y456F). Our data suggest that TSLP-induced STAT5 phosphorylation requires at least one cytoplasmic tyrosine residue in the human TSLP receptor complex. We also examined JAK2 phosphorylation in cells expressing wild type and various mutant receptors and failed to detect any tyrosine phosphorylation induced by TSLP (data not shown) in agreement with previous reports $[2,17,18]$.

\section{Conclusions}

Here we report that mutation of all four cytoplasmic tyrosine residues in the TSLP receptor complex abolishes the proliferative response to TSLP as well as STAT5 phosphorylation. Mutation of individual tyrosine residues in the cytoplasmic domain of human IL-7R $\alpha$ or TSLPR was not sufficient to abolish this proliferative response although it did impair the response at low doses of TSLP $(1 \mathrm{ng} / \mathrm{ml}$ and $10 \mathrm{ng} / \mathrm{ml})$. Intriguingly, these mutations led to an increase in the proliferation rate in response to high doses of TSLP $(100 \mathrm{ng} / \mathrm{ml})$. Further experimentation will be required to elucidate the mechanistic basis of the signals that are mediated by 
A

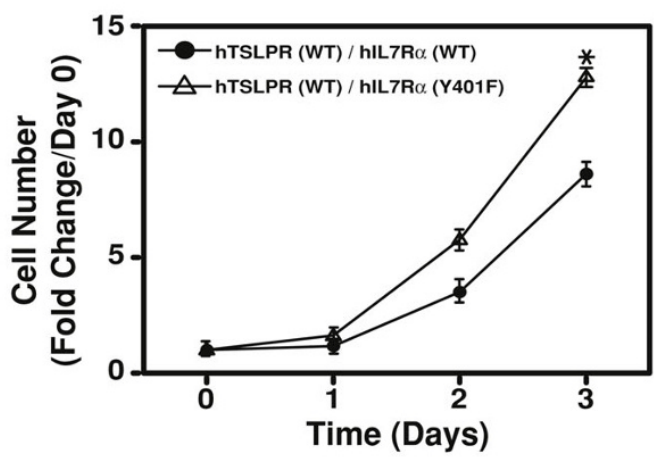

C

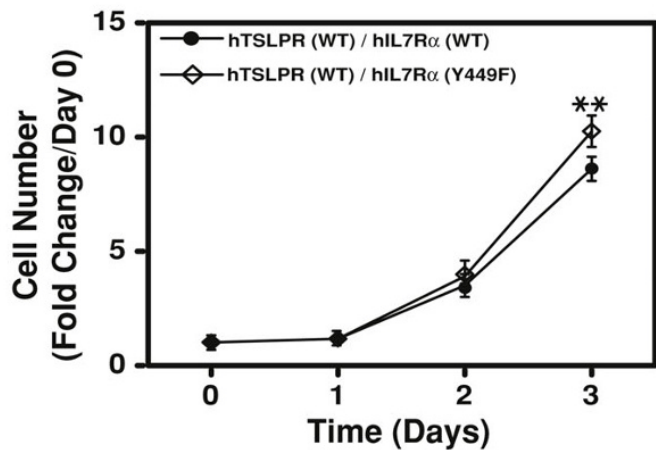

E

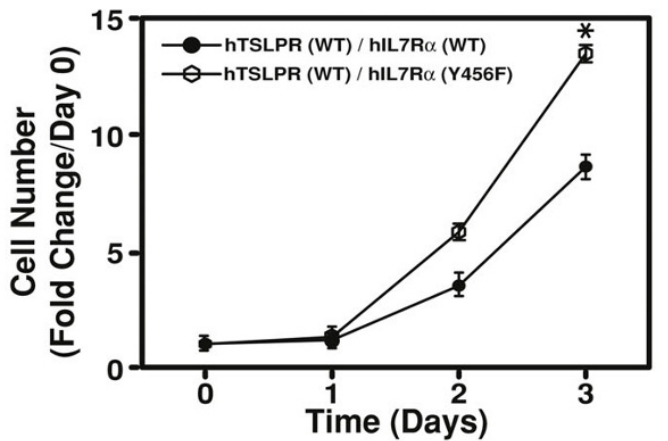

G

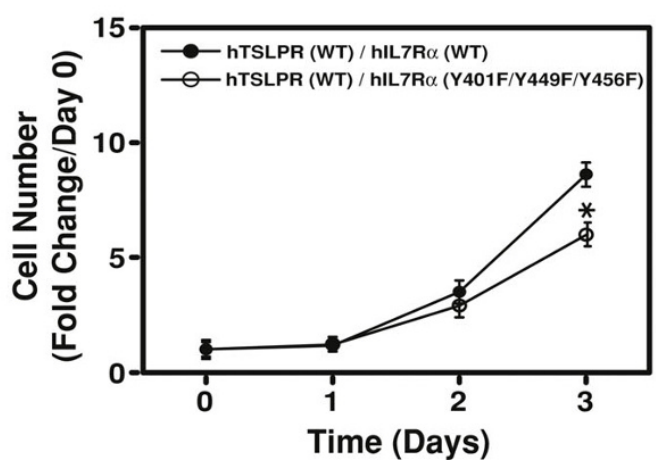

B

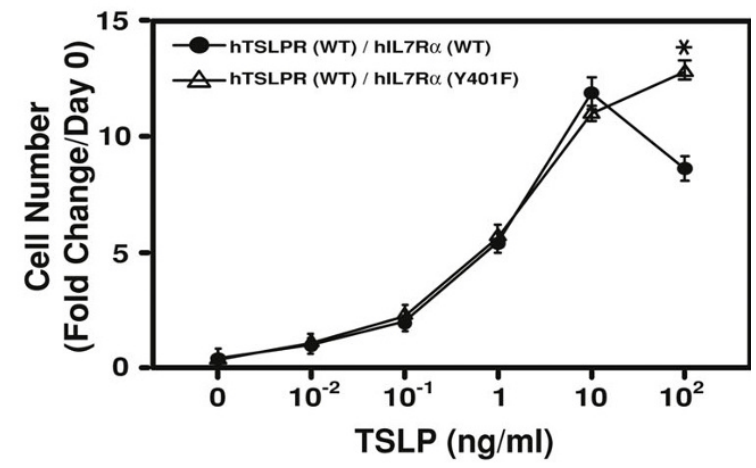

D

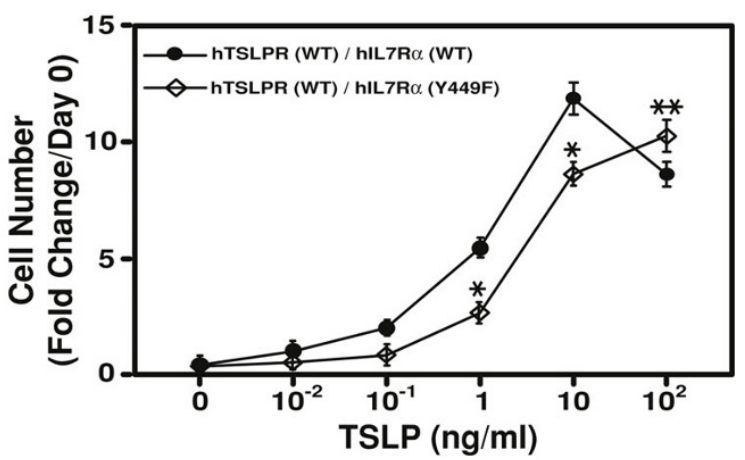

$\mathbf{F}$

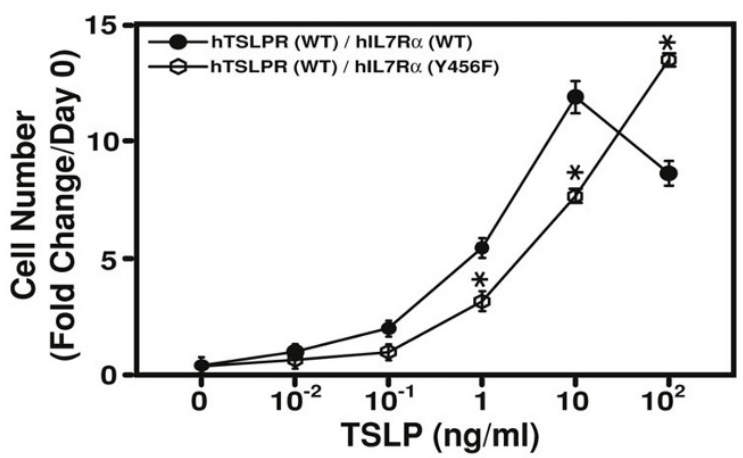

H

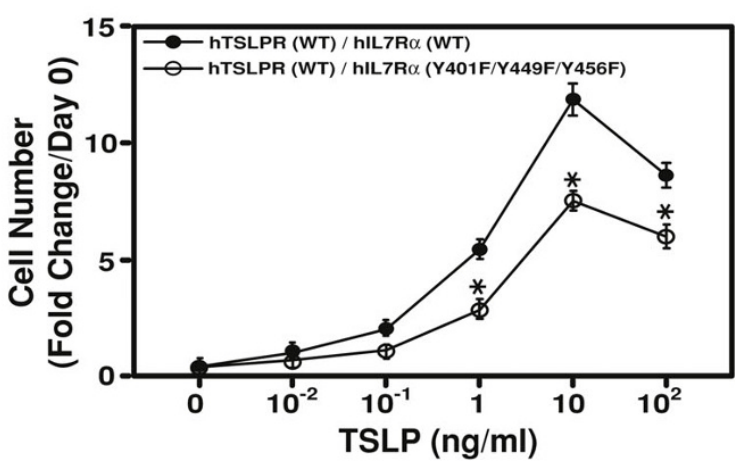

Figure 3 The cytoplasmic tyrosine residues of the human IL-7R $\alpha$ are not required for TSLP-dependent cell proliferation. TSLP-induced proliferation of $\mathrm{Ba} / \mathrm{F3}$ cells co-expressing the indicated receptors was examined as described in Figure 2D. The proliferative response to different doses of TSLP was assessed in Ba/F3 cells expressing the indicated receptors as described in Figure 2E. Statistically significance was calculated using Student's t-test ( ${ }^{*}$ indicates a significant difference with $p$-value $<0.01 ;{ }^{* *} p$-value $<0.05$ ). (The growth of cells was examined in 3 independent sets of experiments and found to be similar. The results from one representative experiment are shown). 
A

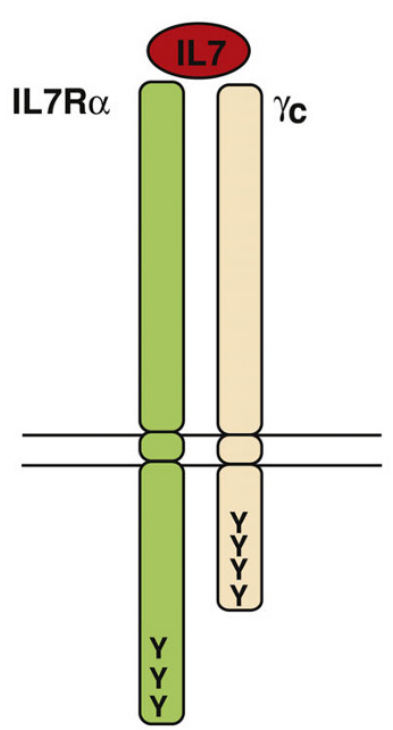

B

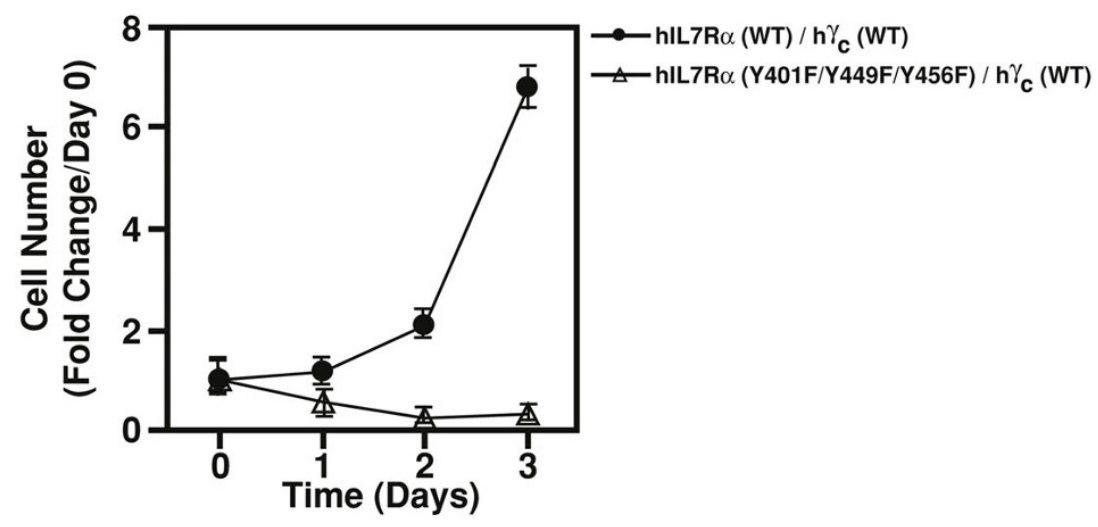

C

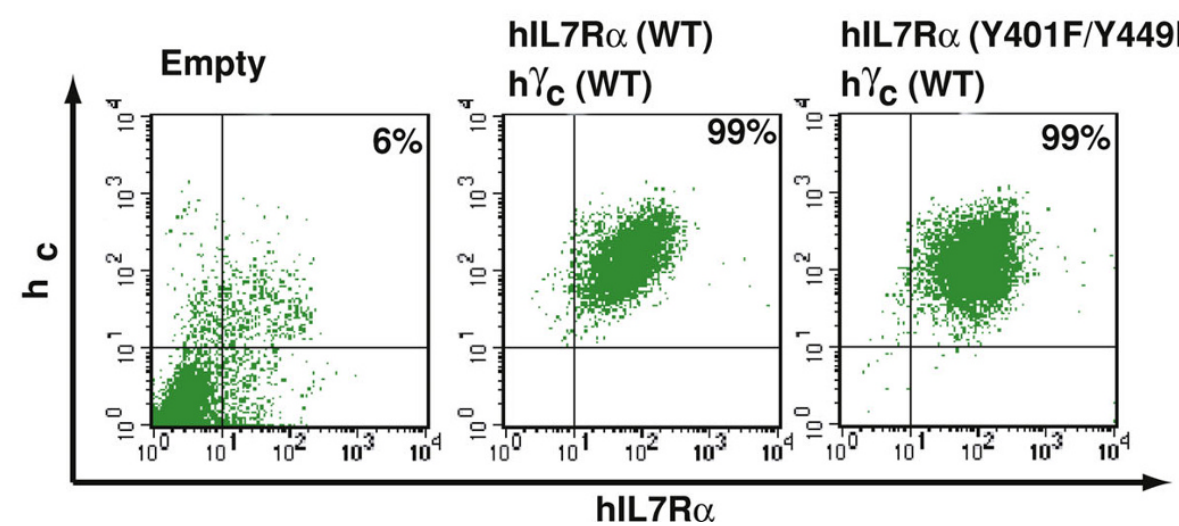

Figure 4 The cytoplasmic tyrosine residues of the human IL-7R $\alpha$ are required for IL-7-dependent cell proliferation. (A) A schematic illustration of the human IL-7 receptor complex composed of the human IL-7R $\alpha$ and the human $\gamma_{c}$. Y denotes the cytoplasmic tyrosine residues. (B) IL-7-induced proliferation of various cell lines as indicated was examined as described in Fig. 2B. $100 \mathrm{ng} / \mathrm{ml} \mathrm{IL-7} \mathrm{was} \mathrm{used.} \mathrm{(C)} \mathrm{Surface}$ expression of the wild type/mutated human IL-7R $\alpha$ and human $\gamma_{c}$ in the cell lines. Exponentially growing infected Ba/F3 cells were analyzed by flow cytometry for cell surface expression of human IL-7R $\alpha$ and $\gamma_{c}$. (The growth of cells was examined in 3 independent sets of experiments and found to be similar. The results from one representative experiment are shown). 
A

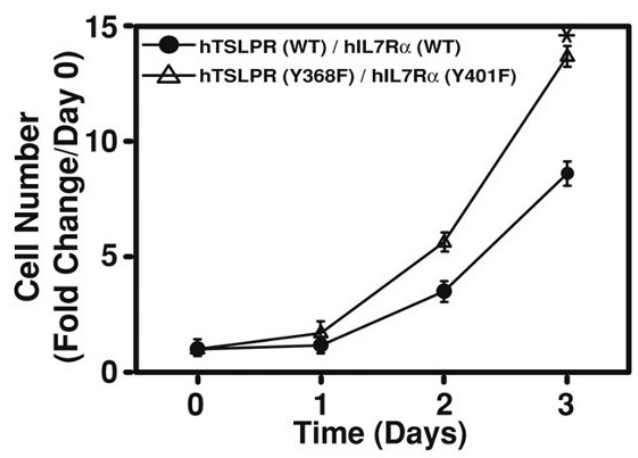

C

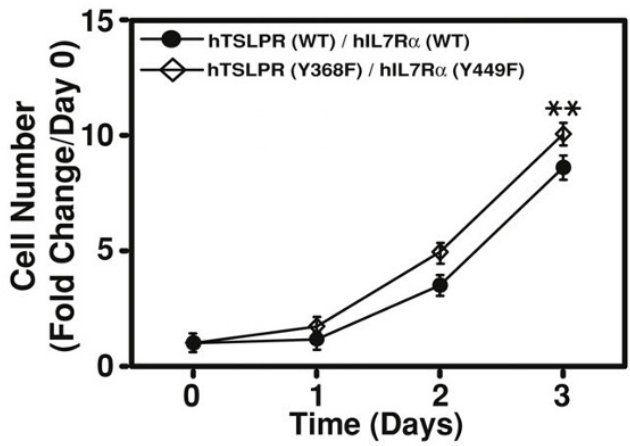

E

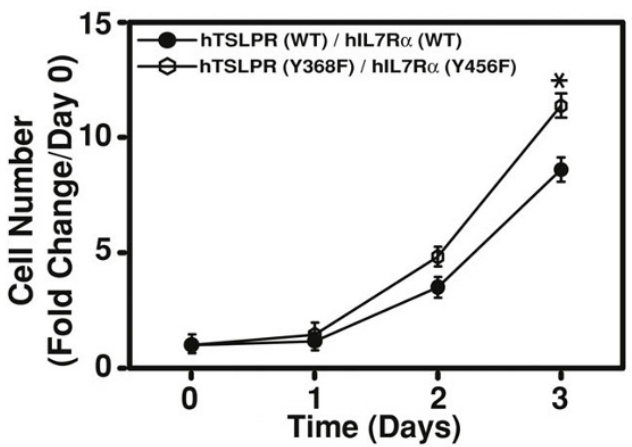

G

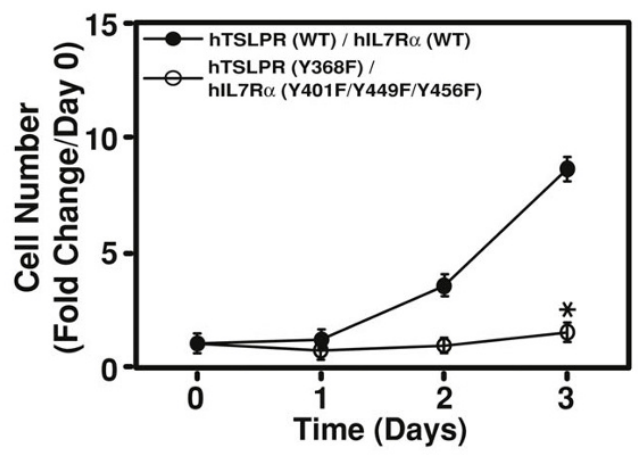

B

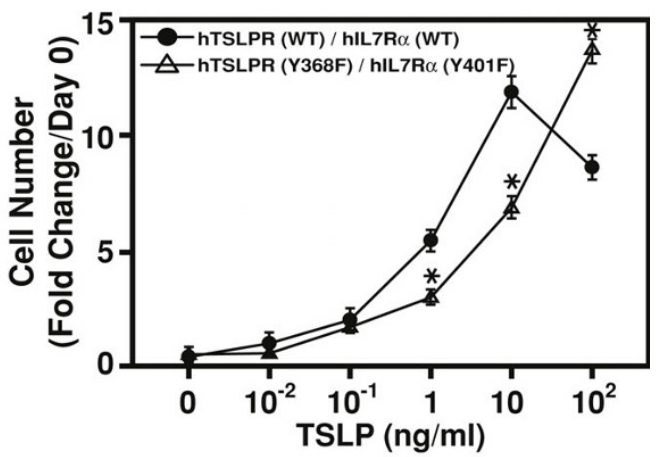

D

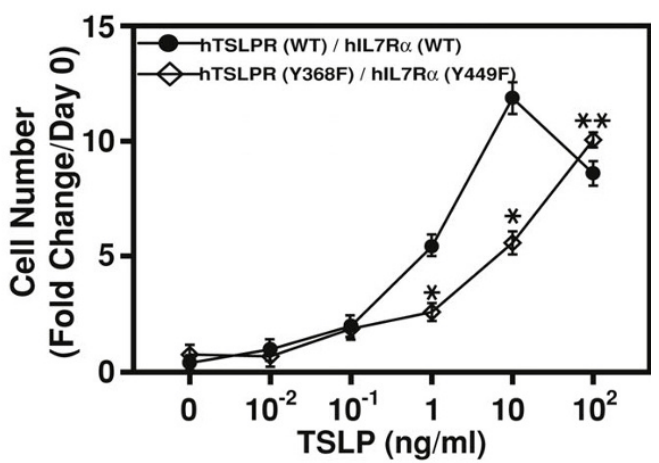

$\mathbf{F}$

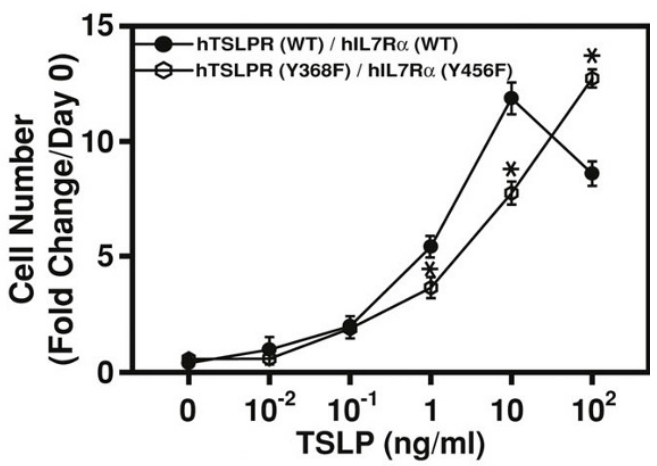

H

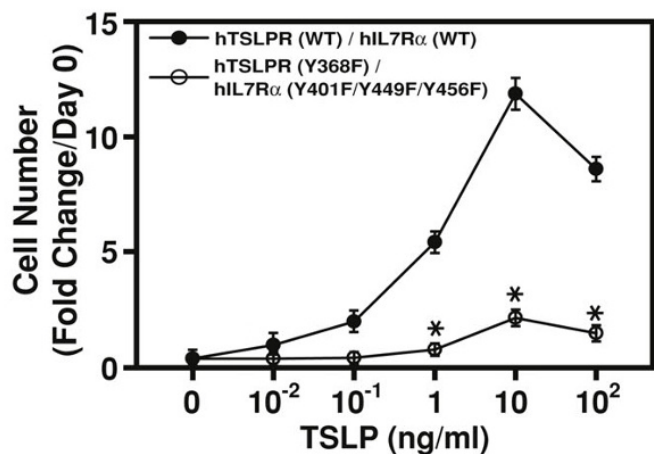

Figure 5 TSLP-dependent cell proliferation requires the presence of at least one tyrosine residue. TSLP-induced proliferation of Ba/F3 cells co-expressing the indicated receptors was examined as described in Figure 2D. The proliferative response to different doses of TSLP was assessed in Ba/F3 cells expressing the indicated receptors as described in Figure 2E. Statistically significance was calculated using Student's t-test ( ${ }^{*}$ indicates a significant difference with $p$-value $<0.01 ;{ }^{* *} p$-value $<0.05$ ). (The growth of cells was examined in 3 independent sets of experiments and found to be similar. The results from one representative experiment are shown). 

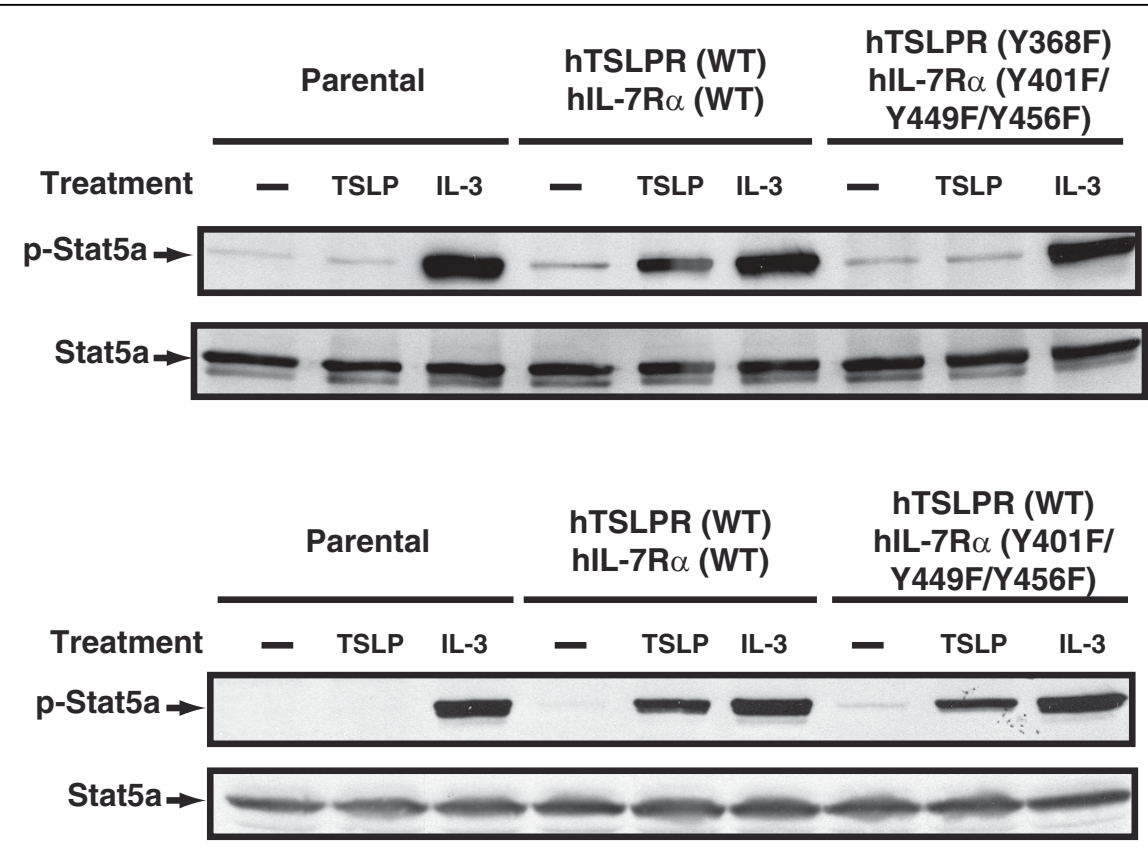

Figure 6 TSLP-induced STAT5 phosphorylation requires the presence of at least one cytoplasmic tyrosine residue. Exponentially growing Ba/F3 cells expressing the indicated receptors were washed three times with RPMI 1640, deprived of IL-3, and then left untreated or stimulated with recombinant human TSLP or mouse IL-3 as shown. After cell lysis, the cell lysates were subjected to immunoprecipitation using anti-STAT5a antibodies. After washing, the immunoprecipitates were resolved by SDS-PAGE. The phosphorylation status of STAT5a was assayed by Western blotting with anti-phosphotyrosine antibodies (4G10) and the total amount of Stat5a was measured by reprobing with anti-Stat5a antibodies as indicated.

the TSLP receptor complex. In this regard, it is interesting to note that the cytosolic tyrosine residues in IL7R $\alpha$ seem to be dispensable for TSLP, but not IL-7, signaling.

\section{Methods Reagents}

PE-conjugated streptavidin, PE-conjugated anti-human CD4, PE-conjugated anti-human $\gamma_{c}$, and Alexa Fluor ${ }^{\circ} 647$ conjugated anti-human IL-7R $\alpha$ antibodies were from BD Biosciences Pharmingen (San Jose, CA, USA). Antiphosphotyrosine antibodies (HRP-conjugated) were from Millipore (Billerica, MA, USA) and anti-Stat5a antibodies were from Santa Cruz Biotechnology (Santa Cruz, CA, USA). Recombinant mouse IL-3, recombinant human IL-7, recombinant human TSLP and biotinylated anti-human TSLPR antibodies were from R\&D Systems (Minneapolis, MN). RPMI 1640, fetal bovine serum (FBS), L-glutamine, and antibiotics were purchased from Invitrogen (Carlsbad, CA, USA). QuikChange XL-II mutagenesis kit was from Stratagene (La Jolla, CA, USA). Sequencing services were performed by the DNA and Peptide Synthesis and Sequencing facility at Johns Hopkins University School of Medicine using dye terminator chemistry. All other reagents used in this study were from Fisher Scientific (Pittsburgh, PA, USA).

\section{Cell culture}

The interleukin-3 (IL-3)-dependent pre B cell line, $\mathrm{Ba} / \mathrm{F} 3$, was grown in RPMI 1640 supplemented with FBS, L-glutamine, penicillin, streptomycin, and mouse recombinant IL-3 $(10 \mathrm{ng} / \mathrm{ml})$. Stably transfected Ba/F3 cell lines were grown in RPMI 1640 supplemented with heat-inactivated FBS, L-glutamine, penicillin, streptomycin and mouse recombinant IL-3 $(10 \mathrm{ng} / \mathrm{ml})$. Human embryonic kidney 293T (HEK293T) cells were cultured in Dulbecco modified essential medium, high glucose, supplemented with FBS, L-glutamine, penicillin, and streptomycin. Cell lines were maintained in the exponential growth phase unless indicated otherwise.

\section{Plasmids and expression vectors}

The human TSLPR was obtained from Dr. James Ihle [19]. The human IL-7R $\alpha$ was subcloned into a bicistronic retrovirus vector PMX-IRES-GFP [20] that expresses GFP while the human TSLPR and $\gamma_{c}$ were subcloned into a bicistronic retrovirus vector pMX-IRES-hCD4 [20] that expresses the human CD4 antigen. The human IL-7R $\alpha, \gamma_{c}$ and TSLPR mutants were generated by sitedirected mutagenesis and confirmed by sequencing.

Generation of stable cell lines using retroviruses

HEK293T cells were transfected with the retroviral vector constructs $(12 \mu \mathrm{g})$ together with the helper virus pCL-ECO $(12 \mu \mathrm{g})$ (Imegenex, San Diego, CA, USA) by 
using Lipofectamine 2000 (Invitrogen). Twenty-four hours after transfection, the supernatants were harvested and filtered through a $0.45-\mu \mathrm{m}$ filter. $\mathrm{Ba} / \mathrm{F} 3$ cells were infected with the pairs of the human IL-7R $\alpha$ in pMXIRES-GFP and the human TSLPR or $\gamma_{c}$ in pMX-IREShCD4. 48 hours after infection, Ba/F3 cells were stained by PE-conjugated anti-human CD4 antibodies and then sorted for GFP and the human CD4 expression.

\section{Receptors cell surface expression}

After sorting, exponentially growing $\mathrm{Ba} / \mathrm{F} 3$ cells expressing the pair of the wild type/mutated TSLP receptor complex were washed twice, stained with biotinylated anti-human TSLPR antibodies followed by PE-conjugated streptavidin and Alexa Fluor 647 conjugated antihuman IL-7R $\alpha$ and analyzed by flow cytometry. Similarly, exponentially growing $\mathrm{Ba} / \mathrm{F} 3$ cells expressing the pair of the wild type/mutated IL-7 receptor complex were washed twice, stained with PE-conjugated antihuman $\gamma_{c}$, and Alexa Fluor 647 conjugated anti-human IL-7R $\alpha$ antibodies and analyzed by flow cytometry.

\section{Immunoprecipitation and Western blotting}

Exponentially growing $\mathrm{Ba} / \mathrm{F} 3$ cells expressing different combinations of receptors were washed three times with RPMI 1640, deprived of IL-3 for $16 \mathrm{~h}$, and then left untreated or stimulated with recombinant human TSLP or mouse IL-3 for 10 minutes at $37^{\circ} \mathrm{C}$. Cells were lyzed in modified RIPA buffer $(50 \mathrm{mM}$ Tris- $\mathrm{HCl}, \mathrm{pH} 7.4,150$ $\mathrm{mM} \mathrm{NaCl}, 1 \mathrm{mM}$ EDTA, $1 \%$ Nonidet P-40, $0.25 \%$ sodium deoxycholate, and $1 \mathrm{mM}$ sodium orthovanadate in the presence of protease inhibitors) followed by centrifugation. The supernatant was subjected to immunoprecipitation using anti-Stat5a antibodies. After washing, the immunoprecipitates were resolved by SDS-PAGE and assayed by Western blotting with anti-phosphotyrosine antibodies (4G10) followed by reprobing with antiStat5a antibodies.

\section{Growth assays}

The growth of cells expressing different combination of receptors was examined in 3 independent sets of experiments. In each experiment, exponentially growing $\mathrm{Ba} / \mathrm{F} 3$ cells (in triplicate) expressing different combinations of receptors were washed three times with RPMI 1640, deprived of IL-3 for $16 \mathrm{~h}$, and then stimulated with recombinant human TSLP or IL-7. Living cells were counted using a Beckman Coulter Z1 (Beckman Coulter, Fullerton, CA, USA). The results (except where indicated) are expressed as increase in cell number (stimulation [n-fold]) as compared to the number of cells plated on day 0. The mean and S.E.M were calculated for each independent experiment. The results from the three independent experiments were similar in each case and one representative experiment is shown in the figures.

\section{Protein sequence alignments}

The protein sequence alignments were performed using ClustalX version 2.0.10 using the BLOSUM62 matrix.

\section{Statistical analysis}

Data were expressed as mean \pm SEM. Differences were examined by Student's t-test between two groups. $\mathrm{p}<0.05$ was considered significant.

\section{List of abbreviations}

TSLP: thymical stromal lymphopoietin; IL-7R $\alpha$ : interleukin-7 receptor $\alpha$ chain; TSLPR: TSLP receptor; $\gamma_{c}$ : interleukin-2 receptor $\gamma$ chain/common $\gamma$ chain.

\section{Acknowledgements}

We thank Dr. James Ihle for providing the human TSLP receptor and Dr. Xuedong Liu for providing pMX-IRES-GFP and pMX-IRES-hCD4 plasmids. Cell sorting was performed by Lee Blosser and Ada Tam in flow cytometry core facility at Johns Hopkins University School of Medicine.

\section{Authors' contributions}

JZ and AP designed the experiments; JZ performed the experiments; JZ and AP drafted the manuscript. All authors read and approved the final manuscript.

\section{Competing interests}

The authors declare that they have no competing interests.

Received: 20 April 2009

Accepted: 8 February 2010 Published: 8 February 2010

\section{References}

1. Friend SL, Hosier S, Nelson A, Foxworthe D, Williams DE, Farr A: A thymic stromal cell line supports in vitro development of surface $\operatorname{lgM}+B$ cells and produces a novel growth factor affecting $B$ and T lineage cells. Exp Hematol 1994, 22:321-328.

2. Levin SD, Koelling RM, Friend SL, Isaksen DE, Ziegler SF, Perlmutter RM, Farr AG: Thymic stromal lymphopoietin: a cytokine that promotes the development of IgM+ B cells in vitro and signals via a novel mechanism. J Immunol 1999, 162:677-683.

3. Huston DP, Liu YJ: Thymic stromal lymphopoietin:a potential therapeutic target for allergy and asthma. Curr Allergy Asthma Rep 2006, 6:372-376.

4. Rochman Y, Leonard WJ: Thymic stromal lymphopoietin: a new cytokine in asthma. Curr Opin Pharmacol 2008, 8:249-254.

5. Pandey A, Ozaki K, Baumann H, Levin SD, Puel A, Farr AG, Ziegler SF, Leonard WJ, Lodish HF: Cloning of a receptor subunit required for signaling by thymic stromal lymphopoietin. Nat Immunol 2000, 1:59-64.

6. Park LS, Martin U, Garka K, Gliniak B, Di Santo JP, Muller W, Largaespada DA, Copeland NG, Jenkins NA, Farr AG, et al: Cloning of the murine thymic stromal lymphopoietin (TSLP) receptor: Formation of a functional heteromeric complex requires interleukin 7 receptor. J Exp Med 2000, 192:659-670.

7. Reche PA, Soumelis V, Gorman DM, Clifford T, Liu M, Travis M, Zurawski SM, Johnston J, Liu YJ, Spits H, et al: Human thymic stromal lymphopoietin preferentially stimulates myeloid cells. J Immunol 2001, 167:336-343.

8. Pallard C, Stegmann AP, van Kleffens T, Smart F, Venkitaraman A, Spits H: Distinct roles of the phosphatidylinositol 3-kinase and STAT5 pathways in IL-7-mediated development of human thymocyte precursors. Immunity 1999, 10:525-535.

9. Corcoran AE, Smart FM, Cowling RJ, Crompton T, Owen MJ, Venkitaraman AR: The interleukin-7 receptor alpha chain transmits distinct signals for proliferation and differentiation during $B$ lymphopoiesis. Embo J 1996, 15:1924-1932.

10. Lin JX, Migone TS, Tsang M, Friedmann M, Weatherbee JA, Zhou L, Yamauchi A, Bloom ET, Mietz J, John S, et al: The role of shared receptor motifs and common Stat proteins in the generation of cytokine 
pleiotropy and redundancy by IL-2, IL-4, IL-7, IL-13, and IL-15. Immunity 1995, 2:331-339.

11. Lai SY, Xu W, Gaffen SL, Liu KD, Longmore GD, Greene WC, Goldsmith MA: The molecular role of the common gamma $\mathrm{c}$ subunit in signal transduction reveals functional asymmetry within multimeric cytokine receptor complexes. Proc Natl Acad Sci USA 1996, 93:231-235.

12. Isaksen DE, Baumann H, Zhou B, Nivollet S, Farr AG, Levin SD, Ziegler SF: Uncoupling of proliferation and Stat5 activation in thymic stromal lymphopoietin-mediated signal transduction. J Immunol 2002, 168:3288-3294.

13. Brown VI, Hulitt J, Fish J, Sheen C, Bruno M, Xu Q, Carroll M, Fang J, Teachey D, Grupp SA: Thymic stromal-derived lymphopoietin induces proliferation of pre-B leukemia and antagonizes MTOR inhibitors, suggesting a role for interleukin-7Ralpha signaling. Cancer Res 2007, 67:9963-9970.

14. Keegan AD, Nelms K, White M, Wang LM, Pierce JH, Paul WE: An IL-4 receptor region containing an insulin receptor motif is important for IL4-mediated IRS-1 phosphorylation and cell growth. Cell 1994, 76:811-820.

15. Demoulin JB, Uyttenhove C, Van Roost E, DeLestre B, Donckers D, Van Snick J, Renauld JC: A single tyrosine of the interleukin-9 (IL-9) receptor is required for STAT activation, antiapoptotic activity, and growth regulation by IL-9. Mol Cell Biol 1996, 16:4710-4716.

16. Zeng R, Spolski R, Casas E, Zhu W, Levy DE, Leonard WJ: The molecular basis of IL-21-mediated proliferation. Blood 2007, 109:4135-4142.

17. Quentmeier H, Drexler HG, Fleckenstein D, Zaborski M, Armstrong A, Sims JE, Lyman SD: Cloning of human thymic stromal lymphopoietin (TSLP) and signaling mechanisms leading to proliferation. Leukemia 2001, 15:1286-1292.

18. Isaksen DE, Baumann $H$, Trobridge PA, Farr AG, Levin SD, Ziegler SF: Requirement for stat5 in thymic stromal lymphopoietin-mediated signal transduction. J Immunol 1999, 163:5971-5977.

19. Carpino N, Thierfelder WE, Chang MS, Saris C, Turner SJ, Ziegler SF, Ihle JN: Absence of an essential role for thymic stromal lymphopoietin receptor in murine B-cell development. Mol Cell Biol 2004, 24:2584-2592.

20. Liu X, Constantinescu SN, Sun Y, Bogan JS, Hirsch D, Weinberg RA, Lodish HF: Generation of mammalian cells stably expressing multiple genes at predetermined levels. Anal Biochem 2000, 280:20-28.

doi:10.1186/1471-2172-11-5

Cite this article as: Zhong and Pandey: Site-directed mutagenesis reveals a unique requirement for tyrosine residues in IL-7R $\alpha$ and TSLPR cytoplasmic domains in TSLP-dependent cell proliferation. BMC Immunology 2010 11:5.

\section{Submit your next manuscript to BioMed Central and take full advantage of:}

- Convenient online submission

- Thorough peer review

- No space constraints or color figure charges

- Immediate publication on acceptance

- Inclusion in PubMed, CAS, Scopus and Google Scholar

- Research which is freely available for redistribution

Submit your manuscript at www.biomedcentral.com/submit
Biomed Central 